\title{
Redistribution of the intra-acrosomal EGFP before acrosomal exocytosis in mouse spermatozoa
}

\author{
Noritaka Hirohashi $^{1,2}$, Florenza A La Spina ${ }^{3}$, Ana Romarowski ${ }^{3}$ and Mariano G Buffone ${ }^{3}$ \\ ${ }^{1}$ Oki Marine Biological Station, Education and Research Center for Biological Resources, Shimane University, \\ 194 Kamo, Okinoshima-cho, Oki, Shimane 685-0024, Japan, ${ }^{2}$ Department of Biological Sciences, Ochanomizu \\ University, 2-1-1 Otsuka, Tokyo 112-8610, Japan and ${ }^{3}$ Instituto de Biología y Medicina Experimental (IBYME), \\ National Research Council of Argentina (CONICET), Vuelta de Obligado 2490 (1428), Buenos Aires, Argentina
}

Correspondence should be addressed to N Hirohashi; Email: hiro@life.shimane-u.ac.jp or to M G Buffone; Email: mgbuffone@ibyme.conicet.gov.ar

\begin{abstract}
Mammalian spermatozoa must undergo complex physiological and morphological alterations within the female reproductive tract before they become fertilization competent. Two important alterations are capacitation and the acrosome reaction (AR), by which spermatozoa become capable of penetrating the zona pellucida (ZP) of the oocyte. Although various biochemical stimulants have been reported to induce the $A R$, the true physiological inducer in vivo remains to be identified. Previously, it has been reported that most fertilizing spermatozoa undergo the AR before contacting the ZP and that only a small fraction of in vitro-capacitated spermatozoa can penetrate the ZP. Therefore, it is important to identify which capacitating spermatozoa undergo the AR in response to potential AR inducers such as progesterone. Here we show that spermatozoa undergo a dynamic rearrangement of the acrosome during in vitro capacitation. This involves the rapid movement of an artificially introduced soluble component of the acrosome, enhanced green fluorescent protein (EGFP), from the acrosomal cap region to the equatorial segment (EQ) of the sperm head. Spermatozoa exhibiting the EQ pattern were more sensitive to progesterone than were those without it. We suggest that spermatozoa that are ready to undergo acrosomal exocytosis can be detected by real-time EGFP imaging. This offers a promising new method for identifying where spermatozoa undergo the AR in the female reproductive tract in vivo.

Reproduction (2015) 149 657-663
\end{abstract}

\section{Introduction}

Mammalian spermatozoa are not able to fertilize oocytes until they have resided in the female reproductive tract for a certain time (Austin 1951, Chang 1951). The changes that occur in spermatozoa during this period are collectively called capacitation. Capacitated spermatozoa must then undergo the acrosome reaction (AR), which is another step before they can pass through the oocyte extracellular matrix called the zona pellucida (ZP) and fuse with the oolemma. It has long been thought that the AR is induced by the contact of the fertilizing spermatozoon with the ZP (Wassarman \& Litscher 2001), although others have reported in hamsters and rabbits that spermatozoa could undergo exocytosis within the cumulus (Yanagimachi 1966, Kuzan et al. 1984). However, recent studies using transgenic mice expressing enhanced green fluorescent protein (EGFP) inside the acrosome as a method of visualizing acrosomal exocytosis revealed that: i) simple sperm binding to the $\mathrm{ZP}$ is not sufficient to induce the AR (Baibakov et al. $2007)$; ii) spermatozoa that have completed the AR are able to bind to the ZP and penetrate it (Jin et al. 2011); and iii) acrosome-reacted spermatozoa collected from the perivitelline space of an oocyte are still able to fertilize other cumulus-enclosed oocytes (Kuzan et al. 1984, Inoue et al. 2011). These observations suggest that fertilizing spermatozoa do not necessarily undergo the AR upon interaction with the ZP proteins in their original three-dimensional structures.

Two obvious questions that have remained unanswered are where and when fertilizing spermatozoa begin their AR in vivo (Bedford 2011, Yanagimachi 2011, Buffone et al. 2014). Although these questions are important, examinations of the AR within oviducts have been difficult. Whereas spermatozoa with intact acrosomes are able to reach the ampullary region of the oviduct following copulation (Chang \& Suarez 2012), acrosome-reacted spermatozoa are present within the oviductal ampulla in vivo before and near the time of fertilization (Yanagimachi 1966, Yanagimachi \& Mahi 1976).

Another unresolved question concerns the real inducer(s) of the AR in fertilizing spermatozoa in vivo. Although the cumulus oophorus surrounding oocytes 
has been implicated as the site of the AR in some mammalian species, such as humans (Stock et al. 1989) and shrews (Kaneko et al. 2001), there is no unequivocal evidence to support this view in mice. In a previous work from our group using IVF, we observed that the $A R$, as shown by a loss of EGFP fluorescence in the acrosome, rarely begins while spermatozoa are traveling through the cumulus (Hirohashi et al. 2011). An inherent problem of using spermatozoa treated in a capacitation medium is that only a small fraction of cells are functionally capacitated, whereas others fail to progress through the steps of sperm-egg interaction, which suggests that the changes that occur in the majority of the spermatozoa do not necessary represent what actually happens during fertilization. This feature may also explain why only a small fraction of the sperm undergoes acrosomal exocytosis when stimulated by progesterone. Therefore, we attempted to identify those spermatozoa whose acrosomes are ready to undergo AR in response to progesterone, one of the major secretory products from the cumulus cells and a potential physiological inducer of the AR (Parinaud et al. 1992, Roldan et al. 1994). It is well established that during capacitation, changes in the acrosome architecture, such as acrosomal swelling, invaginations of the outer acrosomal membrane, and membrane docking, are essential for exocytosis to occur (Zanetti \& Mayorga 2009); however, these changes are difficult to observe using standard microcopy methods. Our hypothesis is that the changes that the acrosome undergoes during capacitation can be visualized using transgenic EGFP sperm, and therefore we might be able to determine spermatozoa whose acrosomes are ready to trigger the AR in response to progesterone. In the present report, we studied the relationship between pre-AR changes in spermatozoa that express EGFP in their acrosomes and their response to progesterone.

\section{Materials and methods}

\section{Materials}

Human tubal fluid (HTF) medium and HEPES-buffered HTF designed for human IVF and ICSI were purchased from InVitroCare (Frederick, MD, USA). BSA and progesterone were obtained from Sigma-Aldrich. Progesterone was dissolved in DMSO for experimental use.

\section{Transgenic mouse spermatozoa}

Spermatozoa of double-gene knock-in male mice (BDF1-Tg (CAG-mtDsRed2, Acr-EGFP) RBGS0020sb) have acrosomes that express EGFP fluorescence and midpiece mitochondria that display Ds-Red2 fluorescence (Hasuwa et al. 2010). The EGFP was designed to be expressed as a soluble protein under the acrosin promoter with a proacrosin signal sequence. These male mice were crossed with imprinting control region strain females, and their gametes were used for our observations.
All experiments were performed with the approval of the Animal Care and Use Committee of Ochanomizu University and IBYME. In addition, investigations using experimental animals were conducted in accordance with the National Institutes of Health specific guidelines (Guide for the Care and Use of Laboratory Animals 1996).

\section{Sperm capacitation}

Spermatozoa recovered from the cauda epididymidis were induced to capacitate by being suspended in a $100 \mu \mathrm{l}$ droplet of HTF-BSA medium at $\sim 10^{5}$ cells $/ \mathrm{ml}$ and being incubated for $1-4 \mathrm{~h}$ at $37^{\circ} \mathrm{C}$ under a humidified atmosphere of $5 \% \mathrm{CO}_{2}$ and $95 \%$ air. In some of the experiments, spermatozoa were incubated with $2 \mathrm{mg} / \mathrm{ml}$ propidium iodide to determine the viability of the cells, and they were observed using fluorescence microscopy.

\section{Analysis of the AR using EGFP-expressing spermatozoa}

To determine the percentage of spermatozoa with EGFP in their acrosomes, $10 \mu \mathrm{l}$ aliquots of the sperm suspensions were placed on poly-L-lysine-coated glass slides and covered with coverslips. To detect the presence of EGFP in the acrosomes, the cells were quantified using a Nikon TE2000 inverted fluorescence microscope with fluorescence optics (excitation: $480 \mathrm{~nm}$; emission: $515 \mathrm{~nm}$ ), as previously described (Buffone et al. 2009a,b). In other experiments, AR was quantified as previously described by flow cytometry using a FACSCanto II flow cytometer (BD) (Muro et al. 2012). The viable sperm were selected by staining with propidium iodide (final concentration: $10 \mu \mathrm{g} / \mathrm{ml}$ ), and their acrosomal integrity was determined by the presence of acrosomal EGFP, as previously reported (Muro et al. 2012).

\section{Real-time observations of acrosomal EGFP}

Spermatozoa were collected as described earlier in the report. Coverslips were washed overnight with $85 \%$ ethanol, rinsed with water, and coated with laminin by adding a drop of $20 \mathrm{mg} / \mathrm{ml}$ laminin (Sigma-Aldrich) and allowing them to air dry. The laminin-covered coverslips were placed into a Leiden chamber (Medical Systems, Greenvale, NY, USA). Spermatozoa were affixed to these coverslips for fluorescence imaging of acrosomal EGFP. The coverslips were then washed twice with $400 \mu \mathrm{l}$ of HTF to remove nonattached spermatozoa. The chamber was placed onto the temperature-controlled stage of an inverted epifluorescence Nikon TE2000 microscope at $37^{\circ} \mathrm{C}$ that was equipped with a $100 \times 0.5-1.3$ NA S Fluor oil objective and a Princeton Instruments MicroMAX CCD camera (Roper Scientific, Trenton, NJ, USA). Basal sperm fluorescence levels were recorded, and - without interrupting the imaging - a small volume of DMSO containing progesterone (Sigma-Aldrich) was added to the coverslip with the spermatozoa and incubated for $30 \mathrm{~s}$ at $37^{\circ} \mathrm{C}$ for a final concentration of $0-20 \mu \mathrm{M}$ progesterone. For analysis, we selected spermatozoa that were oriented such that the side of the head could be visualized and the apical, dorsal, and posterior regions of the sperm head (equatorial segment (EQ)) could be identified readily. The fluorescence 

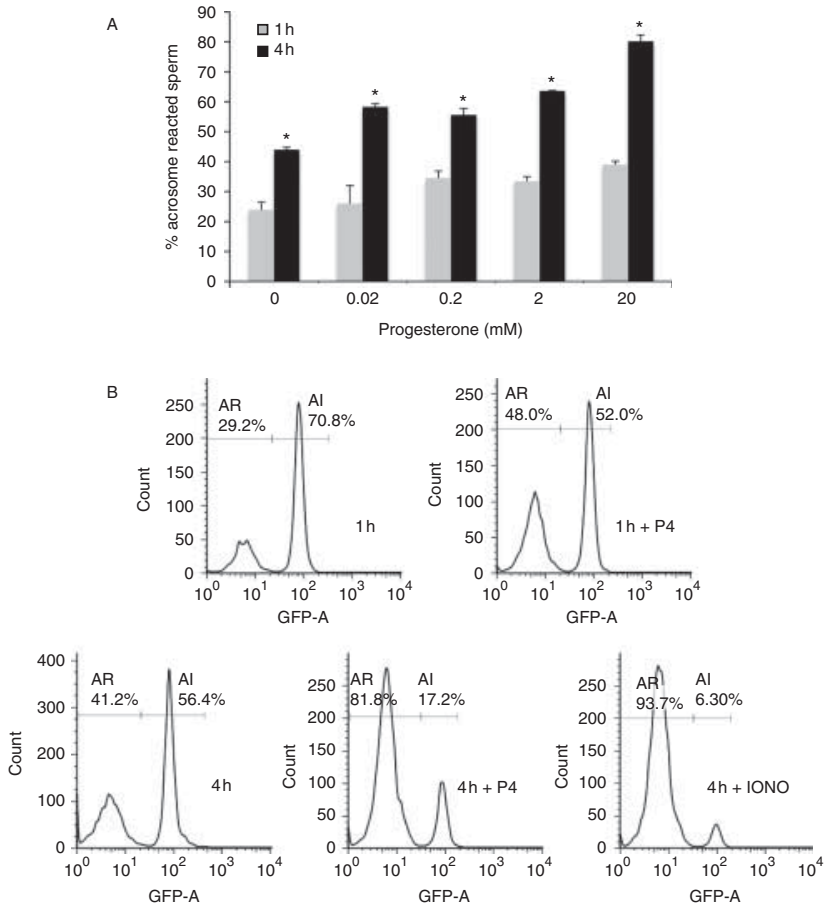

Figure 1 Spermatozoa are more responsive to progesterone after $4 \mathrm{~h}$ incubation in capacitating conditions (as compared to 1 h). (A) After 1 or $4 \mathrm{~h}$ incubation under capacitating conditions, the spermatozoa were exposed to increasing concentrations of progesterone $(0.01-20 \mu \mathrm{M})$ and the percentages of sperm with or without enhanced green fluorescent protein (EGFP) in the acrosome were observed and counted using a fluorescence microscope. As a control, the vehicle (DMSO) was added to the samples at both time points ( $0 \mu \mathrm{M}$ of progesterone). The data represent the mean \pm s.E.M. ( $n=4$ experiments). ${ }^{*}$ Represents significant difference as compared to $1 \mathrm{~h}(P<0.05)$. (B) Representative experiments showing acrosomal exocytosis after progesterone treatment at 1 or $4 \mathrm{~h}$ incubation under capacitating conditions. Live spermatozoa as judged by propidium iodide staining were used independently to determine the percentage of sperm showing the presence $(\mathrm{Al})$ or absence (AR) of the green fluorescent protein (GFP) fluorescence using flow cytometry. After $1 \mathrm{~h}$ incubation in a capacitating medium, sperm were treated with or without $20 \mu \mathrm{M}$ progesterone (upper right and left panel respectively). After $4 \mathrm{~h}$ incubation in a capacitating medium, spermatozoa were treated with or without $20 \mu \mathrm{M}$ progesterone or $1 \mu \mathrm{M}$ ionomycin (middle, left and right panel respectively).

signal was measured every $0.5 \mathrm{~s}$ for $10 \mathrm{~min}$. The fluorescence intensity was quantified using Image Software version $1.47 \mathrm{~V}$ (National Institutes of Health, Bethesda, MD, USA). The intensity of fluorescence was calculated in the regions of interest localized in the EQ. The background intensity was subtracted.

In some experiments, a confocal microscope (Nikon Eclipse C1) was used to determine the localization of EGFP in spermatozoa during the capacitating incubation.

\section{Observation of the sperm acrosomal status in the oviduct}

$(\mathrm{C} 57 \mathrm{BL} / 6 \mathrm{~J} \times \mathrm{BALB} / \mathrm{C}) \mathrm{F} 1$ females were superovulated by i.p. injection of $5 \mathrm{U}$ of pregnant mare's serum gonadotropin followed $48 \mathrm{~h}$ later by $5 \mathrm{U}$ of human chorionic gonadotropin (hCG). Superovulated females were caged together with CAG$m t D s R e d 2, A c r-E G F P$ males $12 \mathrm{~h}$ after hCG injection. At 1 and $4 \mathrm{~h}$ after coitus, the oviducts and uteruses of the females were dissected out. Oviducts were gently manipulated and straightened out by cutting the mesosalpinx. They were mounted on slides, covered with coverslips, and examined by confocal microscopy (Nikon Eclipse C1) to determine the presence of sperm containing the acrosomal EGFP marker.

\section{Statistical analysis}

Data are expressed as mean \pm S.E.M. of at least three experiments for all determinations. Statistical analyses were performed by ANOVA and a Tukey's multiple comparisons test using the GraphPad Prism 6 Software (La Jolla, CA, USA). Statistical significance is indicated in the figure legends.

\section{Results}

Progesterone has long been a candidate for inducing the AR (Parinaud et al. 1992, Roldan et al. 1994). However, the reasons have remained elusive as to why only a small proportion of capacitated spermatozoa can undergo the AR when they are exposed to progesterone at physiological concentrations. Although most IVF protocols use a 1-2 $\mathrm{h}$ incubation of epididymal spermatozoa in a chemically defined medium for sperm capacitation, we wondered if a longer incubation would result in better outcomes in the progesterone-induced AR. A recent report showed that mice spermatozoa remain in the lower part of the oviduct for about $3 \mathrm{~h}$ before ascending to the ampulla (Miki \& Clapham 2013). We therefore studied how spermatozoa would respond to progesterone after various times of in vitro capacitation (1-4 h) with different concentrations of progesterone $(0-20 \mu \mathrm{M})$ (Fig. 1).
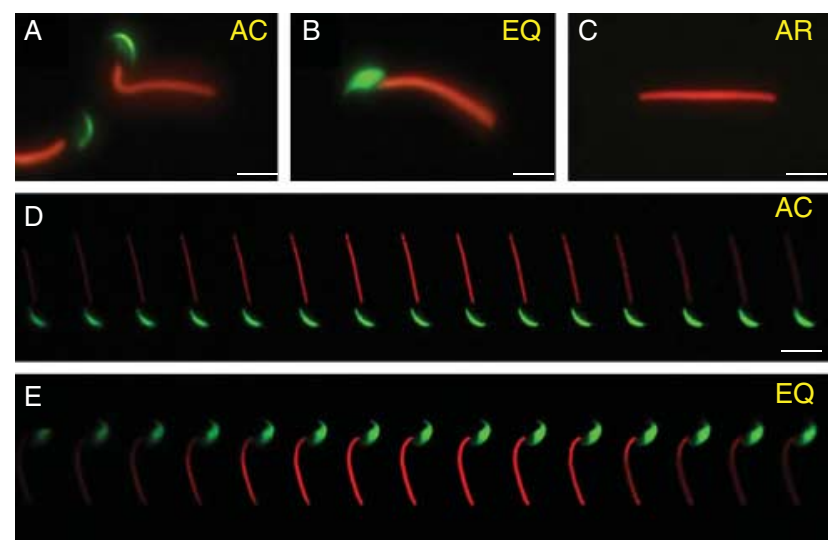

Figure 2 Two different types of acrosome-intact spermatozoa: (A) acrosomal cap (AC) and (B) equatorial segment (EQ). (C) Acrosomereacted sperm lacking enhanced green fluorescent protein (EGFP). (D and E) Z-stack confocal images of AC (D) and EQ (E) spermatozoa respectively. After $4 \mathrm{~h}$ of incubation, many spermatozoa showed expansion of the soluble acrosomal components, which are represented by EGFP presence in the equatorial region. 

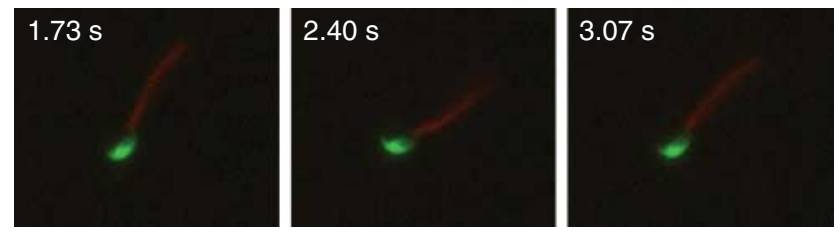

Figure 3 Representative time-lapse images of an equatorial segment (EQ) spermatozoon exhibiting active motility and no membrane permeability to propidium iodide. Images are taken from the Supplementary Movie S1.

The results showed a dose-dependent increase in the progesterone-induced $A R$, and the rate of the increase was accelerated when it was initiated following a $4 \mathrm{~h}$ incubation period as compared to a $1 \mathrm{~h}$ incubation period (Fig. 1). Thus, the ability of spermatozoa to undergo the $A R$ in response to progesterone was enhanced after extended incubation under capacitating conditions.

When the spermatozoa from transgenic mice (Ho et al. 2009) were incubated for $4 \mathrm{~h}$ and observed by epifluorescence microscopy, we noted that a significant number of acrosome-intact spermatozoa exhibited an altered EGFP distribution. That is, the EGFP fluorescence originally restricted to the acrosomal cap region (Fig. 2A) was extended to the EQ of the acrosome (Fig. 2B), hereinafter referred to as EQ sperm, which are distinguishable from noncapacitated, acrosome-intact spermatozoa, which have a distinct acrosomal cap staining pattern (AC sperm). The difference in EGFP distribution between $\mathrm{AC}$ and EQ spermatozoa was not caused by a difference in the focal plane of the microscope, because we observed the same difference when using a confocal microscope (Fig. 2D and E). The EQ sperm were alive and motile, as judged by propidium iodide vital staining and video motion analysis (Fig. 3, which represents Supplementary Movie S1, see section on supplementary data given at the end of this article).

Population analysis of the spermatozoa revealed that the transition from the AC to the EQ pattern occurred gradually during $4 \mathrm{~h}$ of incubation (Fig. $4 \mathrm{~A}$ ). In a control experiment where BSA was absent in the medium (noncapacitating conditions), the transition was seldom seen, which suggests its association with sperm capacitation (Fig. 4B). Interestingly, single-cell analysis revealed that the transition from the $\mathrm{AC}$ to the $\mathrm{EQ}$ pattern occurred very rapidly (in $\sim 10 \mathrm{~s}$ ) (Fig. 5, Supplementary Movie S2, see section on supplementary data given at the end of this article).

We speculated that the change in the EGFP distribution pattern might reflect an important physiological consequence during sperm capacitation before the $A R$, which renders spermatozoa more susceptible to progesterone. When spermatozoa were treated with $20 \mu \mathrm{M}$ progesterone after a $4 \mathrm{~h}$ incubation (Fig. 6A, Supplementary Movies S3 and S4, see section on supplementary data given at the end of this article), both $A C$ and $E Q$ sperm underwent the AR. However, the induced $A R$ occurred to a greater extent in EQ spermatozoa than it did in $A C$ spermatozoa (Fig. 6B).

Next we addressed if sperm can evoke this change after being deposited in the female reproductive tract by copulation. At $4 \mathrm{~h}$ post-mating, the WT females were killed in order to isolate the reproductive organs (uterus and oviduct). Ejaculated spermatozoa in the isolated organs were observed using epifluorescence or confocal microscopy. As shown in Fig. 7A, spermatozoa migrated through the utero-tubal junction (UTJ) and took up residence in the oviduct. The sperm density in the UTJ and in the lower parts of the isthmus (close to the UTJ) was much higher than that in other parts of the oviduct, and it was markedly decreased in the upper part (toward the ampulla) of the fallopian tube.
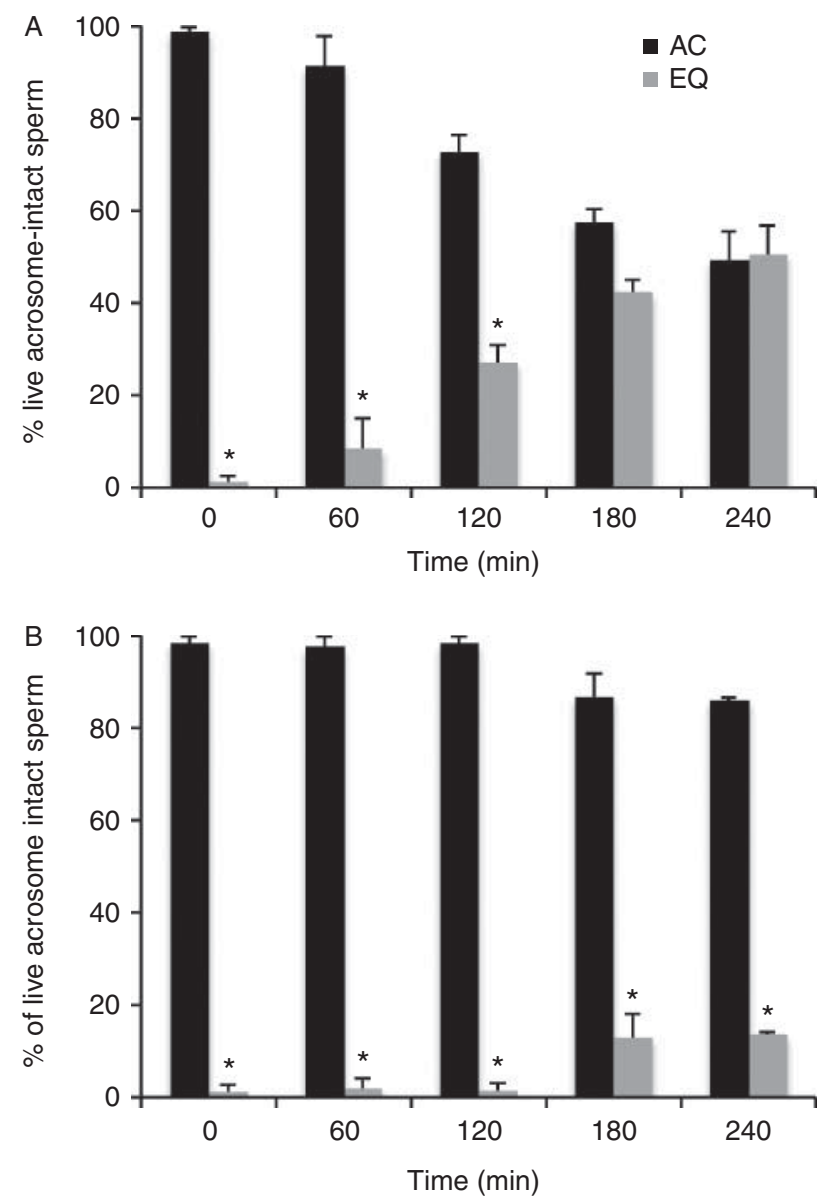

Figure 4 The transition from the acrosomal cap (AC) pattern to the equatorial segment (EQ) pattern depended on capacitating conditions. Time course analysis of the AC (black columns) and EQ (gray columns) patterns: (A) under capacitating conditions, and (B) under noncapacitating conditions. Incubation of spermatozoa in medium lacking bovine serum albumin (BSA) did not permit the transition. Data represent the mean \pm S.E.M. ( $n=4$ experiments). *Represents significant difference compared with AC pattern $(P<0.05)$. 

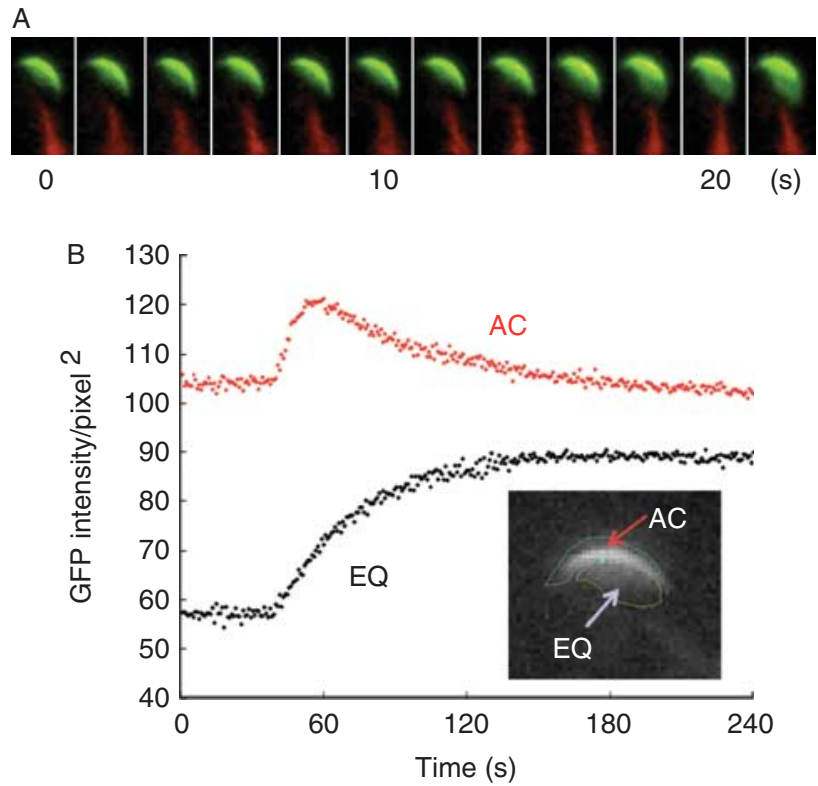

Figure 5 Live imaging analysis of the transition between the acrosomal cap (AC) and the equatorial segment (EQ) patterns. (A) Representative time-lapse images of a spermatozoon undergoing this transition. (B) The fluorescence intensity in the equatorial region area became stably higher after the transition, whereas the fluorescence in the acrosomal cap region increased transiently and returned to the original level upon the AC to EQ transition. The fluorescence intensities of two arbitrary delimited areas of interest were measured over time.

According to a previous report (Ho et al. 2009), spermatozoa arrived at the isthmus $60-90 \mathrm{~min}$ after mating. After $4 \mathrm{~h}$, the sperm density in each section of the oviduct was quite similar to the distribution observed after $1 \mathrm{~h}$ (data not shown). We were unable to find spermatozoa with the EQ pattern in the uterus or the UTJ (data not shown); however, some EQ sperm were evident in the upper segments of the isthmus only after $4 \mathrm{~h}$ post-mating (Fig. 7B and C). However, an important limitation of accurately quantifying the percentage of EQ sperm in this region is that very few cells arrive at this segment of the oviduct, and some of them have already lost their acrosomes once they do so.

\section{Discussion}

In mammals, many millions of spermatozoa are ejaculated into the vagina or the uterus, yet only very few can reach the site of fertilization (the ampulla). An extreme case reported in mice was that the spermto-egg ratio was nearly $1: 1$ in the ampulla (StewartSavage \& Bavister 1988), which suggests that every single sperm that arrived at the fertilization site has gained fertilization competence. However, the mechanism by which how such few spermatozoa are selectively transported has been a long-standing mystery. In contrast, conventional IVF requires thousands of excess capacitated spermatozoa to ensure fertilization.
In standard IVF protocols, epididymal sperm are subjected to a minimum of $1 \mathrm{~h}$ incubation in a chemically defined capacitating medium, and thereafter only $10-20 \%$ of the population can undergo the AR by inducers such as ZP3 or progesterone. These results can draw a tentative conclusion that in vitro-capacitated spermatozoa exhibit heterogeneous fertilizability because of chronological or susceptibility differences in acquiring competence for exocytosis. Meanwhile, we realized during our IVF attempts (Hirohashi et al. 2011, Jin et al. 2011) that mice spermatozoa that were incubated for $4-5 \mathrm{~h}$ in a capacitating medium gave better fertilization outcomes than $1 \mathrm{~h}$ incubated spermatozoa did. This observation prompted us to examine the morphology of the acrosome in great detail during in vitro capacitation. Because the rate of spontaneous AR does not correlate with fertilization outcomes (data not shown) and because fertilization readily occurs if the cumulus oophorus remains intact, fertilizing spermatozoa are thought to have intact acrosomes during capacitation and to undergo the AR upon stimulation by some soluble or insoluble component(s) from the cumulus matrix.

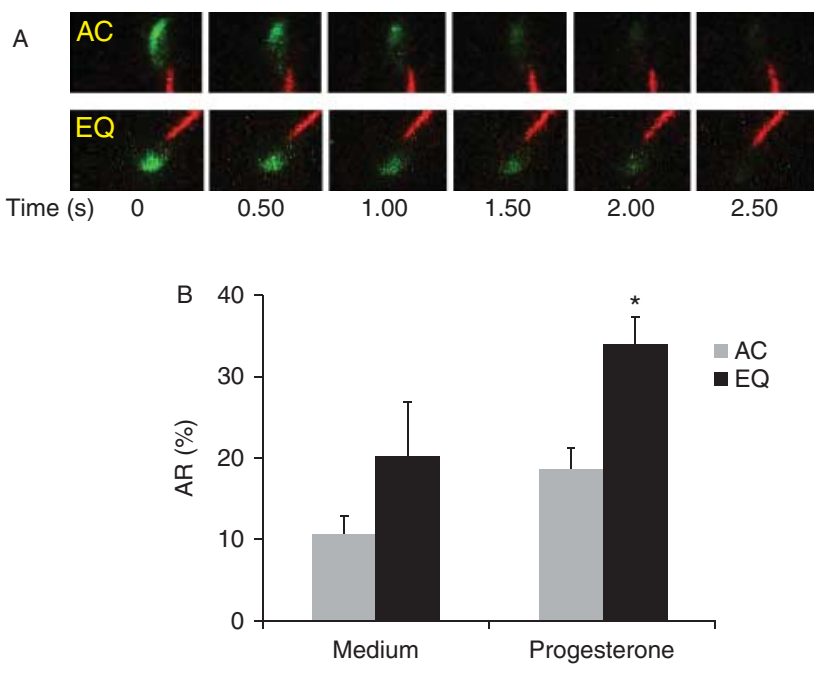

Figure 6 Spermatozoa with both patterns of enhanced green fluorescent protein (EGFP) distribution could undergo the acrosome reaction (AR) after the addition of progesterone. (A) Time-lapse images of EGFP-expressing spermatozoa with the acrosomal cap (AC) pattern (upper panels) or the equatorial segment (EQ) pattern (lower panels) undergoing acrosomal exocytosis after the addition of $20 \mu \mathrm{M}$ of progesterone. (B) The percentages of ARs of both AC- and EQ-type spermatozoa in the presence (induced $A R$ ) or absence (spontaneous $\mathrm{AR}$ ) of $20 \mu \mathrm{M}$ of progesterone. As a control, the vehicle dimethyl sulfoxide (DMSO) was added to the samples (Medium). Spermatozoa were attached to laminin-coated slides and recorded for $10 \mathrm{~min}$. During this time, both patterns underwent spontaneous ARs at similar rates. However, in the presence of $20 \mu \mathrm{M}$ progesterone, more EQ sperm than AC sperm tended to undergo exocytosis. Data represent the mean \pm s.E.M. $(n=4$ experiments). *Represents significant difference as compared to AC pattern $(P<0.05)$. 

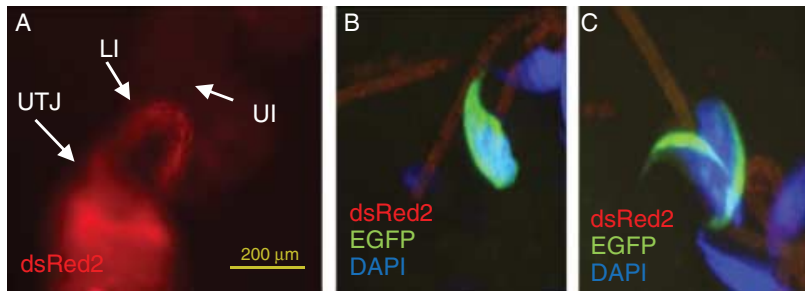

Figure 7 Representative image of sperm inside the female reproductive tract after natural mating with transgenic males carrying Acr-EGFP and Ds-Red2. The photographs show spermatozoa migrating through the female reproductive tract detected by Ds-Red2 using an epifluorescence microscope (A, B and C). Representative confocal images of a cross-section of the upper isthmus isolated $4 \mathrm{~h}$ after mating containing equatorial segment (EQ) (B) and equatorial segment (AC) (C) spermatozoon. UTJ, utero-tubal junction; LI, lower isthmus; UI, upper isthmus.

Interestingly, we observed an increased rate of the AR after a $4 \mathrm{~h}$ of preincubation in capacitating condition as compared to a 1-2 $\mathrm{h}$ preincubation. In the extended incubation condition, there was a dynamic and rapid redistribution of acrosomal EGFP. The EGFP protein expanded from the apical region of the head (acrosomal crescent) to the equatorial domain in a substantial proportion of spermatozoa. Although spermatozoa with either AC or EQ patterns were capable of triggering the $A R$ when they were exposed to progesterone, the EQ spermatozoa underwent the AR more frequently. This change might be related to acrosomal swelling (Zanetti \& Mayorga 2009), which is a prerequisite to the acrosomal exocytosis that occurs following sperm capacitation. According to Zanetti \& Mayorga (2009), human spermatozoa increase their acrosomal volume during capacitation, and this is of critical importance for membrane docking and hybrid vesicle formation during the AR. It remains unknown how acrosomal swelling occurs during sperm capacitation; however, the alkalization of intra-acrosomal milieu could be a possible mechanism (isotonic swelling). Initially, acrosomal $\mathrm{pH}$ is maintained at acidic levels through the homeostatic action of a vacuolar-type proton ATPase (V-ATPase), a sodium-proton exchanger (NHE), and possibly a $\mathrm{Cl}^{-} / \mathrm{HCO}_{3}^{-}$exchanger (Nishigaki et al. 2014). Upon stimulation with a capacitating medium, acrosomal $\mathrm{pH}$ gradually increases over the incubation period of $120 \mathrm{~min}$ from $\mathrm{pH} 5.3 \pm 0.1$ to $\mathrm{pH} \quad 6.2 \pm 0.3$ (Nakanishi et al. 2001). However, because the rate of $\mathrm{pH}$ increase is so gradual, alkalosis-induced swelling does not simply explain the observed dynamics of AC-to-EQ transition; rather, the transition must be set by a commitment threshold.

To investigate the physiological role of the capacitation-associated EGFP distribution, we investigated the acrosomal status of spermatozoa in the uterus and different parts of the oviduct. We observed some EQ sperm in the upper segments of the oviduct. However, because of the limited sperm number arriving at this region, it was difficult to estimate the accurate AC/EQ proportion. In addition, we met difficulty distinguishing the EQ from the AC in some oviductal spermatozoa that preclude an equatorial view. When we compared the sperm density in each part of the oviduct, there was no significant difference between 1 and $4 \mathrm{~h}$ post-mating except for the upper segments of the oviduct. A marked difference was the absence of EQ sperm at any part of the oviduct at $1 \mathrm{~h}$ post-mating. Therefore, EQ sperm could be found at the primed state where the AR takes place by physiological stimulation in the vicinity of the ampulla.

Transition from a nonexcitable (AC) to an excitable (EQ) state might also be a rapid process that leads to acquired exocytotic competence and to an increased sensitivity to progesterone. In the present paper, we provide for the first time a non-invasive method by which live cell imaging of capacitated spermatozoa with an exocytotic competence could be performed. These findings are important not only for dissecting the molecular events that occur before the AR but also for identifying bona fide inducer(s) for the $A R$ from the natural environment of female reproductive organs.

\section{Supplementary data}

This is linked to the online version of the paper at http://dx.doi. org/10.1530/REP-15-0017.

\section{Declaration of interest}

The authors declare that there is no conflict of interest that could be perceived as prejudicing the impartiality of the research reported.

\section{Funding}

This work was supported by the National Institutes of Health (grant number RO1TW008662), the Agencia Nacional de Promoción Científica y Tecnológica (PICT 2010-2426 and 2012-1175), and the Japanese Society for Promotion of Science (JSPS) (FY2011).

\section{Acknowledgements}

We thank Drs Ryuzo Yanagimachi and George L Gerton for their insightful comments. We also thank Dr Masaru Okabe for kindly providing the 'green-red' sperm mice (CAG-mtDsRed2, Acr-EGFP). We thank $\mathrm{N}$ Osafune, M Takada, $\mathrm{H}$ Ochi, and $\mathrm{K}$ Moriwaki for their assistance in the experiments.

\section{References}

Austin CR 1951 Observations on the penetration of the sperm in the mammalian egg. Australian Journal of Scientific Research. Ser. B: Biological Sciences 4 581-596. 
Baibakov B, Gauthier L, Talbot P, Rankin TL \& Dean J 2007 Sperm binding to the zona pellucida is not sufficient to induce acrosome exocytosis. Development 134 933-943. (doi:10.1242/dev.02752)

Bedford JM 2011 Site of the mammalian sperm physiological acrosome reaction. PNAS 108 4703-4704. (doi:10.1073/pnas.1102296108)

Buffone MG, Rodriguez-Miranda E, Storey BT \& Gerton GL 2009a Acrosomal exocytosis of mouse sperm progresses in a consistent direction in response to zona pellucida. Journal of Cellular Physiology 220 611-620. (doi:10.1002/jcp.21781)

Buffone MG, Kim K-S, Doak BJ, Rodriguez-Miranda E \& Gerton GL 2009b Functional consequences of cleavage, dissociation and exocytotic release of ZP3R, a C4BP-related protein, from the mouse sperm acrosomal matrix. Journal of Cell Science 122 3153-3160. (doi:10. 1242/jcs.052977)

Buffone MG, Hirohashi N \& Gerton GL 2014 Unresolved questions concerning mammalian sperm acrosomal exocytosis. Biology of Reproduction 90 112. (doi:10.1095/biolreprod.114.117911)

Chang MC 1951 Fertilizing capacity of spermatozoa deposited into the fallopian tubes. Nature 168 697-698. (doi:10.1038/168697b0)

Chang H \& Suarez SS 2012 Unexpected flagellar movement patterns and epithelial binding behavior of mouse sperm in the oviduct. Biology of Reproduction 86. 140, 1-8. (doi:10.1095/biolreprod.111.096578)

Hasuwa H, Muro Y, Ikawa M, Kato N, Tsujimoto Y \& Okabe M 2010 Transgenic mouse sperm that have green acrosome and red mitochondria allow visualization of sperm and their acrosome reaction in vivo. Experimental Animals/lapanese Association for Laboratory Animal Science 59 105-107. (doi:10.1538/expanim.59.105)

Hirohashi N, Gerton GL \& Buffone MG 2011 Video imaging of the sperm acrosome reaction during in vitro fertilization. Communicative \& Integrative Biology 4 471-476. (doi:10.4161/cib.15636)

Ho K, Wolff CA \& Suarez SS 2009 CatSper-null mutant spermatozoa are unable to ascend beyond the oviductal reservoir. Reproduction, Fertility, and Development 21 345-350. (doi:10.1071/RD08183)

Inoue N, Satouh Y, Ikawa M, Okabe M \& Yanagimachi R 2011 Acrosomereacted mouse spermatozoa recovered from the perivitelline space can fertilize other eggs. PNAS 108 20008-20011. (doi:10.1073/pnas. 1116965108)

Jin M, Fujiwara E, Kakiuchi Y, Okabe M, Satouh Y, Baba SA, Chiba K \& Hirohashi N 2011 Most fertilizing mouse spermatozoa begin their acrosome reaction before contact with the zona pellucida during in vitro fertilization. PNAS 108 4892-4896. (doi:10.1073/pnas.1018 202108)

Kaneko T, lida H, Bedford JM \& Mōri T 2001 Spermatozoa of the shrew, Suncus murinus, undergo the acrosome reaction and then selectively kill cells in penetrating the cumulus oophorus. Biology of Reproduction 65 544-553. (doi:10.1095/biolreprod65.2.544)

Kuzan FB, Fleming AD \& Seidel GE Jr 1984 Successful fertilization in vitro of fresh intact oocytes by perivitelline (acrosome-reacted) spermatozoa of the rabbit. Fertility and Sterility $\mathbf{4 1}$ 766-770.
Miki K \& Clapham DE 2013 Rheotaxis guides mammalian sperm. Current Biology 23 443-452. (doi:10.1016/j.cub.2013.02.007)

Muro Y, Buffone MG, Okabe M \& Gerton GL 2012 Function of the acrosomal matrix: zona pellucida 3 receptor (ZP3R/sp56) is not essential for mouse fertilization. Biology of Reproduction 86 1-6. (doi:10.1095/ biolreprod.111.095877)

Nakanishi T, Ikawa M, Yamada S, Toshimori K \& Okabe M 2001 Alkalinization of acrosome measured by GFP as a pH indicator and its relation to sperm capacitation. Developmental Biology 237 222-231. (doi:10.1006/dbio.2001.0353)

Nishigaki T, José O, González-Cota AL, Romero F, Treviño CL \& Darszon A 2014 Intracellular pH in sperm physiology. Biochemical and Biophysical Research Communication 450 1149-1158. (doi:10.1016/j.bbrc.2014. 05.100)

Parinaud J, Labal B \& Vieitez G 1992 High progesterone concentrations induce acrosome reaction with a low cytotoxic effect. Fertility and Sterility $\mathbf{5 8}$ 599-602.

Roldan ER, Murase T \& Shi QX 1994 Exocytosis in spermatozoa in response to progesterone and zona pellucida. Science 266 1578-1581. (doi:10. 1126/science.7985030)

Stewart-Savage J \& Bavister BD 1988 Success of fertilization in golden hamsters is a function of the relative gamete ratio. Gamete Research $\mathbf{2 1}$ 1-10. (doi:10.1002/mrd.1120210102)

Stock CE, Bates R, Lindsay KS, Edmonds DK \& Fraser LR 1989 Human oocytecumulus complexes stimulate the human acrosome reaction. Journal of Reproduction and Fertility 86 723-730. (doi:10.1530/jrf.0.0860723)

Wassarman PM \& Litscher ES 2001 Towards the molecular basis of sperm and egg interaction during mammalian fertilization. Cells, Tissues, Organs 168 36-45. (doi:10.1159/000016804)

Yanagimachi R \& Mahi CA 1976 The sperm acrosome reaction and fertilization in the guinea-pig: a study in vivo. Journal of Reproduction and Fertility 46 49-54. (doi:10.1530/jrf.0.0460049)

Yanagimachi R 1966 Time and process of sperm penetration into hamster ova in vivo and in vitro. Journal of Reproduction and Fertility 11 359-370. (doi:10.1530/jrf.0.0110359)

Yanagimachi R 2011 Mammalian sperm acrosome reaction: where does it begin before fertilization? Biology of Reproduction 85 4-5. (doi:10.1095/ biolreprod.111.092601)

Zanetti N \& Mayorga LS 2009 Acrosomal swelling and membrane docking are required for hybrid vesicle formation during the human sperm acrosome reaction. Biology of Reproduction 81 396-405. (doi:10.1095/ biolreprod.109.076166)

Received 12 January 2015

First decision 2 February 2015

Revised manuscript received 26 February 2015

Accepted 1 April 2015 